PROCEEDINGS OF THE

AMERICAN MATHEMATICAL SOCIETY

Volume 128, Number 8, Pages 2413-2420

S 0002-9939(00)05622-7

Article electronically published on February 21, 2000

\title{
OPERATOR SEMIGROUPS WITH QUASINILPOTENT COMMUTATORS
}

\author{
HEYDAR RADJAVI, PETER ROSENTHAL, AND VICTOR SHULMAN \\ (Communicated by David R. Larson)
}

\begin{abstract}
It is shown that a multiplicative semigroup of operators is triangularizable if $S T-T S$ is quasinilpotent for every pair $\{S, T\}$ in the semigroup and certain other hypotheses are satisfied.
\end{abstract}

\section{INTRODUCTION AND PRELIMINARIES}

We consider (multiplicative) semigroups of operators on a Hilbert or Banach space. We are interested in those semigroups $\mathcal{S}$ that are commutative "modulo quasinilpotent operators"; i.e., $S T-T S$ is quasinilpotent for all $S$ and $T$ in $\mathcal{S}$. Is such an $\mathcal{S}$ reducible? That is, does there exist a (closed) subspace of $\mathcal{H}$ invariant under all members of $\mathcal{S}$ ? Is $\mathcal{S}$ triangularizable? That is, does the lattice of invariant subspaces of $\mathcal{S}$ contain a maximal chain of subspaces of $\mathcal{H}$ ? Guralnick [1] showed that in finite dimensions the answer is affirmative to both questions. We show that the general answer in infinite dimensions is negative, but there are some positive results in the presence of compactness conditions.

Let $\mathcal{K}(\mathcal{H})$ denote the algebra of compact operators on a Hilbert space $\mathcal{H}$. We shall view $\mathcal{M}_{n}(\mathbb{C})$ as $\mathcal{K}(\mathcal{H})$ where $\mathcal{H}$ is $n$-dimensional. We thank Tom Laffey for a very helpful discussion of group theory.

We shall need the following (known) lemma more than once in the sequel. (The second part of the lemma does not require compactness.)

Lemma 1. A semigroup $\mathcal{S}$ in $\mathcal{K}(\mathcal{H})$ is reducible if it satisfies either of the following conditions:

(a) There is a nonzero continuous linear functional on $\mathcal{K}(\mathcal{H})$ whose restriction to $\mathcal{S}$ is zero.

(b) There is an idempotent $E$ of rank at least two (not necessarily in $S$ ) such that the set of operators (compressions) $E \mathcal{S} E \mid E \mathcal{H}$ is reducible.

Proof. (a) Let $\mathfrak{A}$ be the closed linear span of $\mathcal{S}$. Then $\mathfrak{A}$ is a subalgebra of $\mathcal{K}(\mathcal{H})$ and the functional is zero on $\mathfrak{A}$, by linearity and continuity. If $\mathfrak{A}$ is irreducible, then $\mathfrak{A}=\mathcal{K}(\mathcal{H})$ by Lomonosov's Theorem [5], which is a contradiction.

(b) By using a similarity we can assume $E$ is self-adjoint. By hypothesis, there is a proper sub-projection $F$ of $E$, with $0 \neq F=E F=F E$, whose range is invariant for $E \mathcal{S} E$. This implies $(E-F) S F=0$ for all $S$ in $\mathcal{S}$. If $\mathcal{S} F=0$, then $F \mathcal{H}$ is a

Received by the editors September 23, 1998.

2000 Mathematics Subject Classification. Primary 47A15, 47D03.

(C)2000 American Mathematical Society 
nontrivial invariant subspace for $\mathcal{S}$. Otherwise, the closure of $\mathcal{S F H}$, which is clearly invariant under $\mathcal{S}$, is proper. Thus $\mathcal{S}$ is reducible in either case.

Another result we need is the following lemma, which is a special case of the finiteness lemma of $[9]$.

Lemma $2\left([9)\right.$. Let $\mathcal{P}$ be a property for semigroups $\mathcal{S}$ in $\mathcal{M}_{n}(\mathbb{C})$ such that, whenever $\mathcal{S}$ has property $\mathcal{P}$, then so do:

(a) the semigroup $\mathbb{C} \mathcal{S}$,

(b) the norm closure $\overline{\mathcal{S}}$ of $\mathcal{S}$, and

(c) the semigroup $\hat{\varphi}(\mathcal{S})$ where $\hat{\varphi}$ is any mapping of $\mathcal{M}_{n}(\mathbb{C})$ induced by a field automorphism $\varphi$ of $\mathbb{C}$; i.e., defined entry-wise for a matrix $M$ by $\hat{\varphi}(M)_{i j}=$ $\varphi\left(M_{i j}\right)$. (Note that such a $\hat{\varphi}$ is a ring, but not an algebra, automorphism.)

Assume that $\mathcal{S}$ is a maximal irreducible semigroup with property $\mathcal{P}$ and that $m$ is the minimal nonzero rank of members of $S$. Then $\mathcal{S}$ contains an idempotent $E$ of rank $m$ such that

$$
\operatorname{ESE} \mid \operatorname{Range}(E)=\mathbb{C G},
$$

where $\mathcal{G}$ is a finite group of $m \times m$ matrices.

\section{Finite Dimensions}

Guralnick 1] proved that a semigroup of matrices over any field is triangularizable over an extension field if $S T-T S$ is always nilpotent. Our proof is a little simpler, but it only applies to matrices over the field of complex numbers (or subfields of the complex numbers). Our approach will provide a useful basis for the infinite-dimensional results presented below. We first prove a special case of the main result.

Lemma 3. Let $\mathcal{G}$ be a finite group of unitary matrices such that $U V-V U$ is nilpotent for all $U$ and $V$ in $\mathcal{G}$. Then $\mathcal{G}$ is abelian.

Proof. We use induction on the order of $\mathcal{G}$. Assume, if possible, that $\mathcal{G}$ is a nonabelian group of smallest order satisfying the hypothesis. Then every proper subgroup of $\mathcal{G}$ is abelian. Thus $\mathcal{G}$ is solvable by O.J. Schmidt's Theorem [3, page 280].

As a finite solvable group, $\mathcal{G}$ has a composition series where each factor group is cyclic of prime order. In particular, there is a normal subgroup $\mathcal{G}_{0}$ with $\mathcal{G} / \mathcal{G}_{0}$ of order $p$. Note that, by the minimality of $\mathcal{G}$, this subgroup is abelian and, hence, can be assumed to be contained in the set of diagonal matrices. Pick $G$ in $\mathcal{G} \backslash \mathcal{G}_{0}$. Since $\mathcal{G}$ is generated by $\mathcal{G}_{0}$ and $G$, to complete the proof we need only show that $G$ commutes with every member $H$ of $\mathcal{G}_{0}$. But

$$
G^{-1} H G-H=\left(G^{-1} H\right) G-G\left(G^{-1} H\right)
$$

is nilpotent by hypothesis. Since $\mathcal{G}_{0}$ is normal, $G^{-1} H G-H$ is also diagonal. The only diagonal nilpotent is 0 .

We now present our proof of Guralnick's finite-dimensional result.

Theorem 1 ([1]). Let $\mathcal{S}$ be a semigroup in $\mathcal{M}_{n}(\mathbb{C})$ such that $S T-T S$ is nilpotent for all $S$ and $T$ in $\mathcal{S}$. Then $\mathcal{S}$ is triangularizable. 
Proof. Let us say a semigroup $\mathcal{S}$ has property $\mathcal{P}$ if $S T-T S$ is nilpotent for all $S$ and $T$ in $\mathcal{S}$. Observe that if $E$ is the projection onto an invariant subspace of $\mathcal{S}$, then both $E \mathcal{S} E$ and $(I-E) \mathcal{S}(I-E)$ have property $\mathcal{P}$ if $\mathcal{S}$ does. Thus a simple inductive argument on $n$ would yield the theorem if we show that every $\mathcal{S}$ with property $\mathcal{P}$ is reducible.

Assume, if possible, that $\mathcal{S}$ is irreducible. To obtain a contradiction, we can also assume that $\mathcal{S}$ is maximal relative to $\mathcal{P}$. It is easily checked that $\mathcal{P}$ is stable under scalar multiplication and under induced automorphisms of semigroups, so that the hypotheses (a) and (c) of Lemma 2 are satisfied by $\mathcal{P}$. Also, continuity of spectrum on $\mathcal{M}_{n}(\mathbb{C})$ shows that (b) is satisfied. Thus we obtain $E$ and $\mathcal{G}$ as in the lemma. Since $\mathcal{G}$ is finite, it is similar to a unitary group [12. Thus we can assume, after applying a simultaneous similarity to $\mathcal{S}$, that $\mathcal{G}$ is a group of $m \times m$ unitary matrices. Observe that $E \mathcal{S} E$, and hence $\mathcal{G}$, inherit the property $\mathcal{P}$.

First we consider the case $m>1$. It follows from Lemma 3 that $\mathcal{G}$ is abelian and hence simultaneously diagonalizable. This implies that $E \mathcal{S} E$, when restricted to the range of $E$, is reducible. Hence $\mathcal{S}$ is reducible by Lemma 1, which is a contradiction.

We now assume that $m=1$. We shall show the existence of a nonzero matrix $A$ such that $\operatorname{tr}(A S)=0$ for every $S$ in $\mathcal{S}$; this would contradict the irreducibility of $\mathcal{S}$, by Lemma 1(a). If $\mathcal{S}$ has a member $T$ with $E T \neq 0$ but $E T E=0$, then we can choose $A=E T$. Observe that $E T S-S E T$ is nilpotent by hypothesis and leaves the one-dimensional range of $E$ invariant, by the equation $E T=E T(1-E)$; this implies that

$$
E T S E=E(E T S-S E T) E=0
$$

and thus $\operatorname{tr} E T S=0$. Similarly, if $\mathcal{S}$ has a member $T$ with $T E \neq 0$ but $E T E=0$, we can pick $A=T E$ and use the fact that $T E S-S T E$ is nilpotent. We have to deal with the situation where $T \in \mathcal{S}$ and $E T E=0$ imply $E T=T E=0$.

Pick a $T$ in $\mathcal{S}$ with $(I-E) T E \neq 0$. (Such a member exists by irreducibility.) Since $(T E S) E-E(T E S)$ is nilpotent by hypothesis and its square is easily seen to leave the range of $E$ invariant, we have

$$
(T E S E-E T E S)^{2} E=0, \text { or }
$$

$$
(E T E)(E S(1-E) T E) E S E=0 .
$$

If either $E S E=0$ or $E T E=0$, then, by the preceding paragraph, one of the matrices $E S$ and $T E$ is zero, yielding the equation $E S(1-E) T E=0$. This equation is also obtained from (1) if both $E S E$ and $E T E$ are nonzero. Thus $E S A=0$ for all $S$ in $\mathcal{S}$, with $A=(1-E) T E \neq 0$. It follows that $\operatorname{tr}(S A)=0$, as desired.

The following corollary removes the finiteness and group hypotheses from Lemma 3 , and extends it to normal matrices.

Corollary 1. If $\mathcal{S}$ is a semigroup of normal matrices with $A B-B A$ nilpotent for every pair $\{A, B\}$ in $\mathcal{S}$, then $\mathcal{S}$ is commutative.

Proof. Simultaneously triangularizable normal operators on finite-dimensional spaces are simultaneously diagonalizable. 
Corollary 2. If $\mathcal{S}$ is a compact group of matrices such that $A B-B A$ is nilpotent for all pairs $\{A, B\}$ in $\mathcal{S}$, then $\mathcal{S}$ is abelian.

Proof. Any such group is similar to a unitary group.

Corollary 3. If $\mathcal{S}$ is a self-adjoint semigroup (i.e., $A \in \mathcal{S}$ implies $A^{*} \in \mathcal{S}$ ) such that $A B-B A$ is nilpotent for all $\{A, B\} \subset \mathcal{S}$, then $\mathcal{S}$ is commutative.

Proof. For every $A \in \mathcal{S}, A A^{*}-A^{*} A$ is nilpotent, hence is 0 , so this follows from Corollary 1.

\section{Hilbert SPACE}

Theorem 1 does not hold for general semigroups of bounded operators on $\mathcal{H}$. The following examples show how spectacularly it fails.

(1) Consider the semigroup $\mathcal{S}$ given in 2]. This semigroup is irreducible, and in fact weakly dense, and satisfies $S^{2}=0$ for all $S \in \mathcal{S}$. As follows from [2], $(S T-T S)^{2}=0$ for all pairs $\{S, T\} \subset \mathcal{S}$.

(2) There is even a group $\mathcal{G}$ which is irreducible (in fact, weakly dense in $B(\mathcal{H})$ ) with $A B-B A$ nilpotent for all $A$ and $B$ in $\mathcal{G}$. Just let

$$
\mathcal{G}=\{I+S: S \in \mathfrak{A}\},
$$

where $\mathfrak{A}$ is the algebra generated by the example $\mathcal{S}$ of the preceding paragraph. It was shown in [2] that $\mathfrak{A}$ consists of nilpotents. Products and inverses of members of $\mathcal{G}$ are in $\mathcal{G}$ (the series $(I-S)^{-1}=I+S+S^{2}+\cdots$ has a finite number of nonzero terms), so $\mathcal{G}$ is a group.

Question. If $\mathcal{G}$ is a group in $B(\mathcal{H})$ with $(S T-T S)^{m}=0$ for a fixed $m$ and all $S$ and $T$ in $\mathcal{G}$, is $\mathcal{G}$ reducible?

Note that an affirmative answer in the case $m=1$ would imply that every operator has a non-trivial invariant subspace.

With additional hypotheses we obtain a stronger result in the case $m=1$.

Theorem 2. Let $\mathcal{S}$ be a semigroup of compact operators with at least one nonquasinilpotent member. If $S T-T S$ is quasinilpotent for every pair $S$ and $T$ in $\mathcal{S}$, then $\mathcal{S}$ is reducible.

Proof. We shall assume with no loss of generality that $\mathcal{S}=\mathbb{C S}$. Also, since spectrum is continuous on compact operators, we can assume $\mathcal{S}$ is norm-closed. It follows that $\mathcal{S}$ contains nonzero operators of finite rank. Suppose, if possible, that $\mathcal{S}$ is irreducible. (See the proof of part (a) of Lemma 2 of [11].) It is not hard to see that if $m$ is the minimal positive rank of members of $\mathcal{S}$, then $\mathcal{S}$ contains an idempotent $E$ of rank $m$. (Short proof: pick any member $A$ of rank $m$ in $\mathcal{S}$. Observe that the semigroup $\mathcal{J}=A \mathcal{S} \mid A \mathcal{H}$ is irreducible by Lemma 1 . We also have $\mathbb{C} \mathcal{J}=\mathcal{J}=\overline{\mathcal{J}}$ by our assumptions. Pick a nonzero member $B$ of $\mathcal{J}$ with spectral radius 1 . Then $B$ is similar to a unitary operator [6], [10] and some subsequence of $\left\{B^{n}\right\}$ approaches the $m \times m$ identity.)

We now consider the subsemigroup $E \mathcal{S} E \mid E \mathcal{H}$, viewed as a semigroup of $m \times m$ matrices. By Theorem 1, this semigroup is triangularizable. Thus if $m>1$, it follows from Lemma 1 that $\mathcal{S}$ is reducible. So we can assume $m=1$. This can be dealt with exactly as in the corresponding part of the proof of Theorem 1, yielding reducibility for $\mathcal{S}$. 
Turovskii's beautiful recent theorem 14] states that semigroups of compact quasinilpotent operators are triangularizable, so part of the hypothesis of Theorem 2 is superfluous. In fact, the following stronger theorem holds.

Theorem 3. Let $\mathcal{S}$ be a semigroup of compact operators. If $S T-T S$ is quasinilpotent for every pair $S$ and $T$ in $\mathcal{S}$, then $\mathcal{S}$ is triangularizable.

Proof. Let $\mathcal{E}$ be a chain of subspaces which is maximal in the lattice of all invariant subspaces of $\mathcal{S}$. We must show that if $\mathcal{M}$ and $\mathcal{N}$ are in $\mathcal{E}$, if $\mathcal{M} \subset \mathcal{N}$, and if there is no member of $\mathcal{E}$ between $\mathcal{M}$ and $\mathcal{N}$, then the codimension of $\mathcal{M}$ in $\mathcal{N}$ is no greater than 1 . Let $P$ be the self-adjoint projection whose range is the orthogonal complement of $\mathcal{M}$ in $\mathcal{N}$. Then it can be easily verified that the corresponding compression of $\mathcal{S}$,

$$
P \mathcal{S} P \text { | range } P \text {, }
$$

forms a semigroup satisfying all the hypotheses of the theorem. It follows that it suffices to show, under these hypotheses, that $\mathcal{S}$ is reducible.

If $\mathcal{S}$ contains a non-quasinilpotent member, then it is reducible by Theorem 2 . So assume that every member of $\mathcal{S}$ is quasinilpotent. Then reducibility follows from Theorem 4(ii) of [14].

The following is an immediate consequence.

Corollary 4. If $\mathcal{S}$ is a semigroup satisfying the hypotheses of the preceding theorem, and if $S$ and $T$ are quasinilpotent members of $\mathcal{S}$, then $S+T$ is quasinilpotent.

Note also that Corollaries 1 and 3 above can be established for semigroups of compact operators by the same proofs as were given in the finite-dimensional case. For the next corollaries, the following lemma is required.

Lemma 4. A semigroup of scalar translates of compact operators is triangularizable if every finite subset of it is triangularizable.

Proof. Let $\mathfrak{A}$ be the algebra generated by the set $\mathcal{E}$ of all $K$ such that there is a $\lambda$ with $\lambda+K$ in the semigroup. Every finite subset of $\mathcal{E}$ is triangularizable. If $A$ and $B$ are any two members of $\mathfrak{A}$, then there is a finite subset $\mathcal{F}$ of $\mathcal{E}$ such that $A$ and $B$ are linear combinations of words in members of $\mathcal{F}$. Thus $\{A, B\}$ is triangularizable. It is shown in [5] that an algebra of compact operators is triangularizable if every pair of members of the algebra is triangularizable. Hence $\mathfrak{A}$, and also the semigroup, are triangularizable.

Corollary 5. A semigroup of translates of finite-rank operators is triangularizable if $A B-B A$ is nilpotent for all $A$ and $B$ in the semigroup.

Proof. In view of Lemma 4, it suffices to show that every finite subset of the semigroup is triangularizable. Given $\left\{\lambda_{i}+F_{i}\right\}_{i=1}^{k}$ in the semigroup, let $\mathcal{H}_{0}$ be the span of all the ranges of $\left\{F_{i}\right\}_{a=1}^{k}$ and $\left\{F_{i}^{*}\right\}_{i=1}^{k}$. Clearly, $\mathcal{H}_{0}$ is finite-dimensional, $\mathcal{H}_{0}$ and $\mathcal{H}_{0}^{\perp}$ are invariant under all the $\left\{F_{i}\right\}$, and the restriction of $\lambda_{i}+F_{i}$ to $\mathcal{H}_{0}^{\perp}$ is $\lambda_{i}$ for each $i$. By Theorem 1, the restriction of the semigroup to $\mathcal{H}_{0}$ is triangularizable. Hence the semigroup is triangularizable.

Corollary 6. A semigroup of normal operators that are scalar translates of finite rank operators is abelian if $A B-B A$ is nilpotent for all $A$ and $B$ in the semigroup. 
Proof. Let $A$ and $B$ be in the semigroup. By using the same proof as given for the above corollary, we can write

$$
A=A_{0} \oplus A_{1} \quad \text { and } \quad B=B_{0} \oplus B_{1}
$$

when $A_{0}$ and $B_{0}$ operate on the same finite-dimensional space, and $A_{1}$ and $B_{1}$ are each multiples of the identity. Theorem 1 implies that $\left\{A_{0}, B_{0}\right\}$ is triangularizable, so $A_{0}$ commutes with $B_{0}$ and it follows that $A$ commutes with $B$.

Corollary 7 (11]). A semigroup of compact operators is triangularizable if every pair of operators in the semigroup is triangularizable.

Proof. This follows immediately from Theorem 3, since the triangularizability of $\{A, B\}$ implies that $A B-B A$ is quasinilpotent.

It should be noted that the finite-dimensional case of Corollary 7, which follows from Theorem 1 above, also follows from the much earlier papers [15] and [16].

\section{Compact groups of operators on Banach spaces}

In this section, $X$ denotes a complex Banach space. We show that a strongly compact group of operators which has quasinilpotent commutators must be abelian.

Definition. A vector $x$ is a finite vector for a semigroup $\mathcal{S}$ of bounded linear operators if the linear span of $\{S x: S \in \mathcal{S}\}$ is finite-dimensional.

The following theorem is essentially implicit in most proofs of the Peter-Weyl Theorem.

Theorem 4. If $\mathcal{G}$ is a group of bounded linear operators on a Banach space, and if $\mathcal{G}$ is compact in the strong operator topology, then the set of finite vectors for $\mathcal{G}$ is dense in the space.

Proof. We need some basic facts about the space $C(\mathcal{G})$ of continuous complexvalued functions on $\mathcal{G}$. If $\rho$ is a continuous representation of $\mathcal{G}$ on a finite-dimensional vector space $V, v \in V$ and $\phi \in V^{*}$, then the function $f$ defined by

$$
f(G)=\phi(\rho(G) v)
$$

is a member of $C(\mathcal{G})$, sometimes called a "matrix element". Let $L$ denote the linear span of all such matrix elements (over all $\rho$, all $v$ and all $\phi$ ). Implicit or explicit in most proofs of the Peter-Weyl Theorem is the assertion that $L$ is uniformly dense in $C(\mathcal{G})$. (For example, this follows immediately from Lemmas 7 and 8 on page 147-148 of [4.) We need two additional properties of $L$ :

(1) there is a sequence $\left\{f_{n}\right\} \subset L$ such that

$$
\left\{\int_{\mathcal{G}} f_{n}(G) f(G) d G\right\} \rightarrow f(I)
$$

for all $f \in C(\mathcal{G})$ (where $d G$ is Haar measure on $\mathcal{G}$ ); and

(2) for each $f \in L$ there is an $n$ and functions $\left\{\alpha_{i}\right\}_{i=1}^{n}$ and $\left\{\beta_{i}\right\}_{i=1}^{n}$ in $C(G)$ such that

$$
f(G H)=\sum_{i=1}^{n} \alpha_{i}(G) \beta_{i}(H)
$$

for all $G$ and $H$ in $\mathcal{G}$. 
To prove $(1)$, note that $L^{1}(\mathcal{G})$ has an approximate identity, so there is such a sequence $\left\{f_{n}\right\} \subset L^{1}(\mathcal{G})$. Since $L$ is uniformly dense in $C(\mathcal{G})$, and $C(\mathcal{G})$ is dense in $L^{1}(\mathcal{G})$, the sequence may be taken in $L$.

It suffices to prove (2) for each matrix element. Identifying the finite-dimensional vector space $V$ with its dual we can assume that $\left\{e_{1}, \ldots, e_{k}\right)$ is a basis for $V$ and the matrix element $f$ has the form $f(\cdot)=\left(\rho(\cdot) e_{i}, e_{j}\right)$ for fixed $i, j$. Then $f(G H)=\left(\rho(G H) e_{i}, e_{j}\right)=\left(\rho(G) \rho(H) e_{i}, e_{j}\right)$. Let $\alpha_{l}(G)=\left(\rho(G) e_{l}, e_{i}\right)$ and $\beta_{l}(H)=$ $\left(\rho(H) e_{j}, e_{l}\right)$. Then $f(G H)=\sum_{l=1}^{n} \alpha_{l}(G) \beta_{l}(H)$, proving $(2)$.

We can now explicitly construct a dense set of finite vectors. Starting with any vector $x \in X$ and any $f \in L$, define

$$
x_{f}=\int_{\mathcal{G}} f(G) G x d G .
$$

We claim that $x_{f}$ is a finite vector for $\mathcal{G}$. To see this, let $H \in \mathcal{G}$. Then

$$
\begin{aligned}
H x_{f} & =\int_{\mathcal{G}} f(G) H G x d G \\
& =\int_{\mathcal{G}} f\left(H^{-1} G\right) G x d G \\
& =\sum_{i=1}^{n} \alpha_{i}\left(H^{-1}\right) \int_{\mathcal{G}} \beta_{i}(G) G x d G .
\end{aligned}
$$

Thus for every $H \in \mathcal{G}, H x_{f}$ is in the finite-dimensional space spanned by the vectors $y=\int_{\mathcal{G}} \beta_{k}(G) G x d G$ for $k=1, \ldots, n$, so each $x_{f}$ is a finite vector for $\mathcal{G}$.

All that remains to be shown is that the linear span of $\left\{x_{f}: x \in X, f \in L\right\}$ is dense in $X$. But if $\Phi \in X^{*}$ and $\Phi\left(x_{f}\right)=0$ for all $x$ and all $f$, then, using the sequence $\left\{f_{n}\right\}$ from (1) above,

$$
\Phi\left(x_{f_{n}}\right)=\int_{\mathcal{G}} f_{n}(G) \Phi(G x) d G
$$

converges to $\Phi(x)$. Thus $\Phi\left(x_{f_{n}}\right)=0$ for all $n$ implies $\Phi(x)=0$, and $\Phi$ is identically 0 . Hence the set $\left\{x_{f}: x \in X, f \in L\right\}$ is dense in $X$.

Theorem 5. A strongly compact group of operators on a Banach space is abelian if all its commutators are quasinilpotent.

Proof. Let $\mathcal{G}$ be such a group. By Theorem 4 , the set $X_{0}$ of finite vectors for $\mathcal{G}$ is dense in the space. Fix any $x \in X_{0}$. Then the invariant subspace $M$ of $\mathcal{G}$ generated by $x$ is finite-dimensional. For any $A, B \in \mathcal{G}$, then, $A B x=B A x$ by Corollary 2 above. Since $A B=B A$ on a dense set, it follows that $A B=B A$.

Corollary 8. A compact group contained in a unital Banach algebra is abelian if all its commutators are quasinilpotent.

Proof. This follows immediately from the proceeding theorem applied to the group of operators on the Banach algebra consisting of the left multiplications by the elements of the given group. 


\section{REFERENCES}

[1] R.M. Guralnick, Triangularization of Sets of Matrices,, Lin. Mult. Alg. 9 (1980), 133-140. MR 82d:15009

[2] D. Hadwin, E. Nordgren, M. Radjabalipour, H. Radjavi, and P. Rosenthal, A nil algebra of bounded operators on Hilbert space with semisimple norm closure,, Integ. Eq. Op. Theory 9 (1986), 739-743. MR 87k:47104

[3] B. Huppert, Enliche Gruppen I, Springer-Verlag, Berlin, Heidelberg, New York, 1967. MR 37:302

[4] T. Husain, Introduction to Topological Groups, Saunders, Philadelphia, 1966. MR 34:278

[5] C. Laurie, E. Nordgren, H. Radjavi, and P. Rosenthal, On triangularization of algebras of operators, J. Reine Angew. Math. 327 (1981), 143-155. MR 83d:47014

[6] W.E. Longstaff and H. Radjavi, On permutability and submultiplicativity of spectral radius, Canadian J. Math. 47 (1995), 1007-1022. MR 97d:47005

[7] V.I. Lomonosov, Invariant subspaces for operators commuting with compact operators, Functional Anal. Appl. 7 (1973), 213-214.

[8] E. Nordgren, H. Radjavi, and P. Rosenthal, Triangularizing semigroups of compact operators, Indiana Univ. Math. J. 33 (1984), 271-275. MR 85b:47047

[9] M. Radjabalipour and H. Radjavi, A finiteness lemma, Brauer's theorem, and other irreducibility results, Comm. Algebra 27 (1999), 301-319. CMP 99:07

[10] H. Radjavi, On reducibility of semigroups of compact operators, Indiana Univ. Math. J. 39 (1990), 499-514. MR 91m:47009

[11] H. Radjavi and P. Rosenthal, From local to global triangularization, J. Funct. Anal. 147 (1997), 443-456. MR 98j:47010

[12] J.P. Serre, Linear Representations of Finite Groups, Springer-Verlag, New York, 1977. MR 56:8675

[13] V.S. Shulman, On invariant subspaces of Volterra operators, Funk. Anal. i Prilozhen. 18 (1984), 85-86 (Russian). MR 85g:47008

[14] Yu. V. Turovskii, Volterra semigroups have invariant subspaces, J. Funct. Anal. 162 (1999), 313-322. CMP 99:10

[15] D.B. Wales and H.J. Zassenhaus, On L-groups, Math. Ann. 198 (1972), 1-12. MR 49:2787

[16] H.J. Zassenhaus, On L-semigroups, Math. Ann. 198 (1972), 13-22.

Department of Mathematics, Dalhousie University, Halifax, Nova Scotia, Canada B3H 3J5

E-mail address: radjavi@mscs.dal.ca

Department of Mathematics, University of Toronto, Toronto, Ontario, Canada M5S 3G3

E-mail address: rosent@math.toronto.edu

Department of Mathematics, Vologda Polytechnical Institute, 15 Lenin St., 16008 Vologda, Russia

E-mail address: sev@vgpi.vologda.su 